\title{
High Resolution Microscopic Optical Mapping of Anatomical and Functional Reentries in Human Cardiac Cell Cultures
}

\author{
Andreu M. Climent ${ }^{1}$, Ismael Hernandez-Romero ${ }^{1}$, Maria S Guillem ${ }^{2}$, Nuria Montserrat ${ }^{3}$, \\ Maria Eugenia Fernández ${ }^{1}$, Felipe Atienza ${ }^{1}$, Francisco Fernandez-Aviles ${ }^{1}$ \\ ${ }^{1}$ Hospital General Universitario Gregorio Marañón, Instituto de Investigación \\ Sanitaria Gregorio Marañón, Madrid, Spain \\ ${ }^{2}$ ITACA, Universitat Politècnica de Valencia, Valencia, Spain \\ ${ }^{3}$ Institute for Bioengineering of Catalonia (IBEC), Barcelona, Spain
}

\begin{abstract}
Anatomical and/or functional reentries have been proposed as one of the main mechanism of perpetuation of cardiac fibrillation processes. However, technical limitations have difficult the characterization of those reentries and are hampering the development of effective anti-arrhythmic treatments. The goal of this study is to present a novel technology to map with high resolution the center of fibrillation drivers in order to characterize the mechanisms of reentry. Cell cultures of human cardiac-like cells differentiated from pluripotent stem cells were analyzed with a novel microscopic optical mapping system. The pharmacological response to verapamil administration of each type of reentry was analyzed. In all analyzed cell cultures, a reentry was identified as the mechanism of maintenance of the arrhythmia. Interestingly, the administration of verapamil produced opposite effects on activation rate depending on the mechanisms of reentry (i.e. anatomical or functional).

Microscopic optical mapping of reentries allows the identification of perpetuation mechanisms which has been demonstrated to be linked with different pharmacological response.
\end{abstract}

\section{Introduction}

Recent experimental and clinical results have demonstrated that the perpetuation of cardiac fibrillatory process may be related with micro-anatomical re-entries and/or functional rotors [1]. Rotors are a special type of reentry pattern of activation, termed also functional reentry, as its action potential circulates around an excitable but unexcited core, in contrast to anatomical reentry which pivots around an unexcitable, such as fibrotic or ischaemic region. Despite the fact that both experimental and mathematical models have suggested that fibrillation may be maintained by those reentries, the lack of appropriate research models and technical system to analyse fibrillating cardiac tissue precludes the elucidation of the underlying mechanisms and the identification of appropriated therapeutic targets.

The goal of the present study is to present a novel technology to map with high resolution (500x500um) the centre of fibrillation drivers in order to characterize the mechanisms of reentry.

The potential of this novel mapping system is illustrated by analysing properties of anatomical and functional reentries spontaneously generated in monolayers of human cardiac-like cells. Specifically, human somatic cells, as epicardial fibroblasts, are converted towards induced pluripotent stem cells and then differentiated into cardiac-like cells [2]. In addition, the different response of those reentries to verapamil antiarrhythmic drug is evaluated.

\section{Methods}

Cell cultures of human cardiomyocytes differentiated from pluripotent stem cells were analysed with a novel microscopic optical mapping system. During fibrillation the dominant driver was identified (i.e. anatomical vs. functional reentry) and characterized in terms of its dominant frequency. The pharmacological response to verapamil administration of each type of reentry was analysed.

\subsection{In vitro generation of cardiomyocyte- like cells from human pluripotent stem cells}

Monolayers of human cardiac like cells were generated from pluripotent human cells as previously described [2]. Briefly, episomal plasmids were used to generate human induced pluripotent stem cells lines in a period of only 
20-22 days from fibroblasts. After nucleofection reaction was performed in a provided Amaxa certified cuvette using nucleofection program U-23 from the Nucleofector $^{\mathrm{TM}} 2 \mathrm{~b}$ Device (Amaxa cat.no. AAB-1001), cells were immediately transferred into two wells of a sixwell culture plate with pre-warmed fibroblast culture media and incubated for 4 additional days with daily media change. Finally, nucleofected cells were subcultured onto matrigel (354277, Corning) coated plates in the presence of mTeSR1 (05850, Stem Cell Technologies). On day 23 hiPSC colonies were picked manually and expanded in matrigel (354277, Corning) coated plates in the presence of mTeSR1 (05850, Stem Cell Technologies). From this stage on human induced pluripotent stem cells colonies were amplified by trypsinization in matrigel (354277, Corning).

For the differentiation of pluripotent stem cells into cardiomyocye-like cells, single cell suspension of pluripotent cells were seeded onto matrigel (BD Biosciences) pre-coated cell culture dishes at a density of 125,000 cells per $\mathrm{cm}^{2}$ in mTeSR medium (StemCell Technologies). Cells were then maintained in mTeSR for $48 \mathrm{~h}$. Differentiation was initiated by treatment with 12 mM CHIR99021 (Selleck) in RPMI (Invitrogen) supplemented with B27 minus insulin (Life Technologies), 2mML-glutamine, $\quad 0.1 \mathrm{~m} \quad$ M2mercaptoethanol, nonessential amino acids and penicillinstreptomycin (RPMI/B27-insulin medium) for $24 \mathrm{~h}$ (day 0 to day 1 ). On day 1 , inhibitor was then removed by intensive washing once with RPMI and medium was changed to RPMI/B27-insulin. On day 3, cells were treated with $5 \mathrm{mM}$ Wnt inhibitor IWP4 (Stemgent) in RPMI/B27-insulin medium and cultured without medium change for $48 \mathrm{~h}$. On day 5, cells were washed once with RPMI to eliminate the inhibitor and maintained in RPMI (Invitrogen) supplemented with B27 (Life Technologies), 2mML-glutamine, $0.1 \mathrm{mM}$ 2-mercaptoethanol, nonessential amino acids and penicillin-streptomycin (RPMI/B27 medium). From day 5, cells were maintained in RPMI/B27 medium with medium change every 2 days. On day 14, beating monolayers were obtained.

\subsection{Optical mapping}

Simultaneous voltage and calcium imaging was developed by means of a novel high-resolution mapping system adapted to a modified version of the previously described dual-mapping system [3]. Specifically a customized OLYMPUS MVX10 was adapted to a highspeed an electron- multiplying charge coupled device (EMCCD) camera as recording system (Evolve-128: 128x128, 24x24 $\mu \mathrm{m}$ square pixels, 16 bit; Photometrics, Tucson, AZ). This system allowed the online control of mapping area from $500 \times 500 \mu \mathrm{m}$ to $5 \times 5 \mathrm{~mm}$. We measure transmembrane voltage using di-8-ANEPPS, and intracellular calcium by using Fura-2AM (TEFLabs, Inc,
Austin, TX. USA). A schematic illustration of the spectral distribution of exciting and emission filters is depicted in figure 1.

Custom software written in MATLAB was used to control the system and perform optical mapping image processing. Specifically, during mapping experiments, stable functional and anatomical reentries were identified over time and space by applying phase map analysis at several space resolution analyses (i.e. from $5 \times 5 \mathrm{~mm}$ to $500 \times 500 \mu \mathrm{m})$. Online phase maps of each movie were obtained by calculating the instantaneous phase of the Hilbert transformed optical recordings [4]. The phase signal ranges between 0 and $2 \pi$ represent the relative delay of each signal in one period.

For the identification of anatomical reentries, a singularity point or centre of rotation was defined as the point in a phase map that is surrounded by phases from 0 to $2 \pi$ [4]. These phase transitions were evaluated at three concentric circles centred at each evaluated point. Phase singularities (PS) were defined as points at which the phases in at least two of these three circles were required to fulfil the following two conditions: 1) phase transition between two consecutive pixels not exceeding $0.6 \pi$ and 2) monotonic phase changes. Once all singularity points were identified, they were connected in time and space into rotors.

For the identification of anatomical reentries, nonelectrically active areas were defined as those formed by recorded pixels with an optical signal 20dB lower than the mean surrounding tissue. A non-electrically active area was defined as a functional reentry when the phase signal around it followed a stable pattern of crescent 0 to $2 \pi$ without jumps higher than $0.1 \pi$.

Power spectra of optical signals were estimated by using a Welch periodogram (2-s Hamming window overlap). Dominant frequency (DF) of each pixel was determined as the frequency with the largest peak in the spectrum between 0.05 and $30 \mathrm{~Hz}$. For each individual cell culture, the highest DF was defined as the maximum DF of the entire dish.

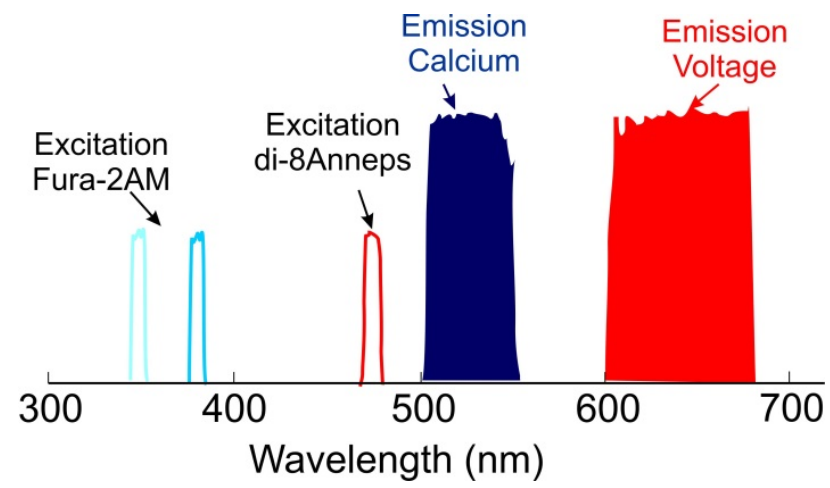

Figure 1. Illustration of the spectral distribution of exciting and emission filters of the simultaneous voltage and calcium mapping. 


\subsection{Statistical analysis}

The previously described analysis was performed in 28 monolayers of human cardiac-like cells. For each monolayer, optical mapping recordings (4s duration) were acquired under basal conditions and after the administration of verapamil ( $4 \mu \mathrm{M}$ in Krebs solution). Data are presented as means \pm SD. Continuous baseline variables were compared using Student's t-test or MannWhitney test, depending on the variable's statistical distribution.

\section{Results}

In 26 of the 28 of the cell cultures (i.e. 92.8\%) electrically functional human cardiac-like cells were mapped. In the remaining 2 cell cultures no electrical activity was identified due to the non-survival of cells.

In all 26 analyzed cell cultures, a stable reentry was identified as the mechanism of maintenance of the arrhythmia. No foci or multiple unstable wavelets were observed in any culture. Microscopic analysis of the reentries allowed their classification into (1) microanatomical $(46 \%, \mathrm{~N}=12)$ or functional reentries $(54 \%$, $\mathrm{N}=14$ ). Isochronal maps of a representative example of each group are shown in the figure 2. Anatomical reentries were present in those cell cultures in which the monolayer was not completely formed, whereas functional reentries appear when a sufficiently large area of the culture did not present any obstacle.

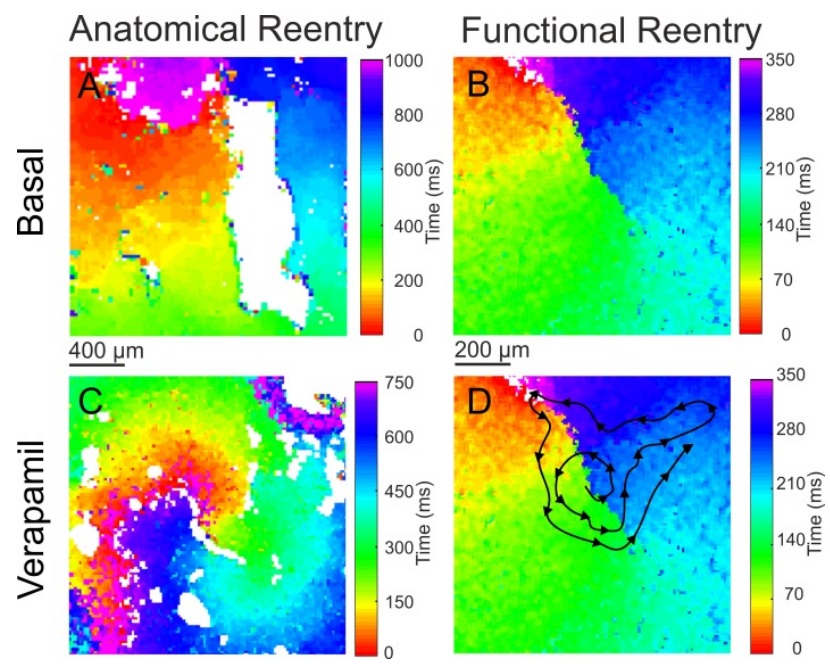

Figure 2. Isochronal maps of two representative examples of anatomical (left) and functional (right) reentries during basal conditions (top) and after the administration of verapamil (bottom). Black line in panel $\mathrm{D}$ indicates the meandering of the functional reentry after the administration of verapamil.

Anatomical reentries presented lower dominant frequencies, and consequently activation times, than functional reentries (i.e. $1.08 \pm 0.19 \mathrm{~Hz}$ vs. $2.96 \pm 0.24 \mathrm{~Hz}$, $\mathrm{p}<0.01$ ) (Figure 3). This was directly related with a longer time needed by an anatomical reentry to complete a loop around an obstacle. In the case of functional reentries, the activation rate was significantly high, since the path required by the wavefront to complete a loop was not limited by any anatomical obstacle, but by the excitability of the tissue.

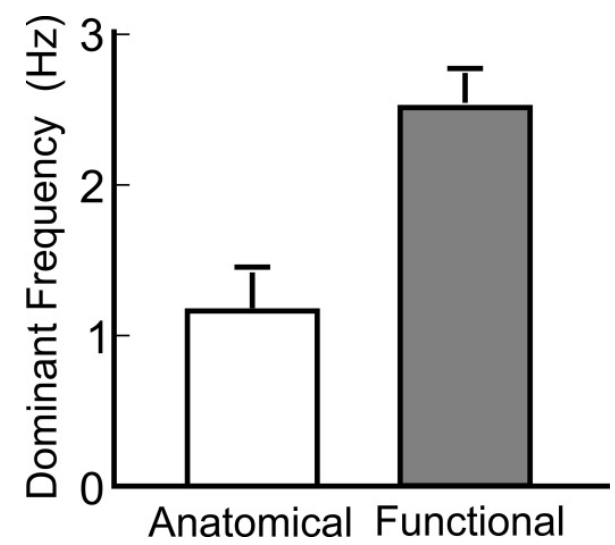

Figure 3. Dominant frequency of anatomical and functional reentries in basal conditions.

\section{Relative variation of Dominant Frequency}

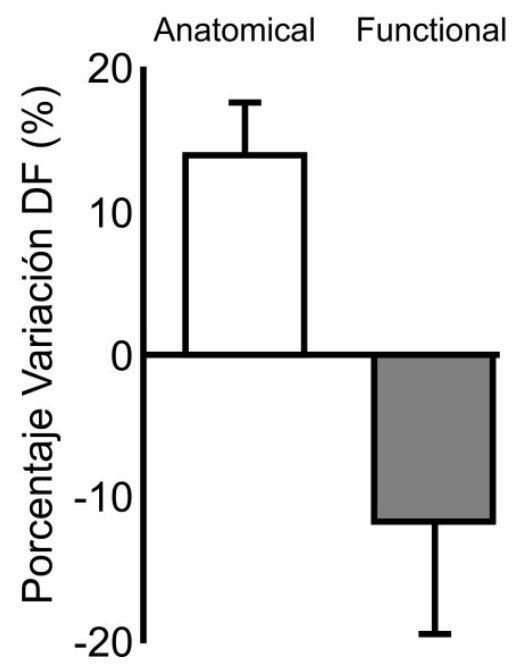

Figure 4. Relative variation of dominant frequency in each group of reentries (i.e. anatomical and functional) after the administration of verapamil.

Interestingly, the administration of verapamil produced opposite effects in each group: whereas DF increased in $15 \pm 3.4 \%$ for anatomical reentries, it decreased in $11.9 \pm 6.8 \%$ for functional rotors $(\mathrm{p}<0.01)$ (Figure 4 ).

The comparative analysis of reentries before and after 
the administration of verapamil allowed us to evaluate the mechanisms that produced the observed opposite effects on the activation rate. For anatomical reentries, administration of verapamil was associated with a change in the anatomical obstacle that maintained the arrhythmia (Figure 2C). Obstacles observed as responsible for the reentry during verapamil were smaller than those identified previously to the drug administration. Those smaller anatomical obstacles required shorter times to complete a loop and consequently produced faster activation rates.

In the case of functional reentries, administration of verapamil was associated with higher movements of the rotor tip while completing a loop (Figure 2D). This increase in the so-called rotor meandering of functional reentries after the administration of verapamil implied that a wavefront needed to complete a longer loop and consequently produced lower activation rates.

\section{Discussion}

In the present study, microscopic analysis of cardiac reentrant mechanisms has been for the first time analyzed thanks to a novel mapping technology. This high resolution mapping technique allowed us to evaluate the effects of a clinically available antiarrhythmic drug (i.e. verapamil) on the mechanisms of fibrillation perpetuation.

Interestingly, our results demonstrated that the effects of the drug are highly dependent on the mechanism of reentry (i.e. anatomical and functional reentries). Whereas in the functional reentries, verapamil produced an increase of the activation rate, which can be considered as proarrhytmic, for functional reentries, verapamil produced a reduction in the activation rate, which can be considered as antiarrhythmic.

Analysis of the high resolution microscopic optical mapping of anatomical and functional reentries before and after the administration of verapamil allows us to suggest the mechanisms for that opposite effect of verapamil. Notice that verapamil is mainly an L-type calcium channel ( $\mathrm{I}_{\mathrm{CaL}}$ ) blocker which in humans may also have a significant effect blocking rapid repolarizing potassium current $\left(\mathrm{I}_{\mathrm{Kr}}\right)$ current. Consequently, the reduction of calcium and potassium currents due to the administration of verapamil is expected to produce a reduction in the duration of the action potential allowing shorten reentries. This is observed in the case of functional reentries. However, for functional reentries, excitability and not action potential duration governs the reentry. Consequently a reduction of excitability due to the reduction of $I_{C a L}$ implied that the reentrant wavefront needed to meander in order to find excitable tissue. This higher rotor meandering produced longer reentrant loops linked with lower activation rates. These results are coherent with the predictions recently offered by mathematical models of human atrial fibrillation [5].

Despite the fact that these novel results have been obtained from in-vitro cell cultures which its intrinsic limitations (e.g. slow conduction velocity, lack of 3D structure), here we made use of last technology for the generation of human cardiac-like cell cultures [2]. Nevertheless, thanks to the fast evolution of tissue engineering technology, we expect that in the near future, the novel technology here presented will be useful to increase the understanding of fibrillation mechanisms and antiarrhythmic treatments.

\section{Conclusion}

High resolution microscopic optical mapping of reentries allows the identification of fibrillation perpetuation mechanisms which has been demonstrated to be linked with different pharmacological response.

\section{Acknowledgements}

Supported in part by grants from the Instituto de Salud Carlos III and Ministry of Economy and Competitiveness of Spain: (Grants PI13-01882, PI13-00903 and PI14/00857, Red RIC, PLE2009-0152 and Red de Terapia Celular); Spanish Society of Cardiology (Grant for Clinical Research in Cardiology 2015); Spanish Ministry of Economy and Competitiveness, Spain (Grant: IJCI-2014-22178 and TEC2013-50391-EXP).

\section{References}

[1] Guillem MS, Climent AM, Rodrigo M, et al. Presence and stability of rotors in atrial fibrillation: evidence and therapeutic implications. Cardiovasc Res. 2016;109:480-92

[2] Garreta E, de Oñate L, Fernández-Santos ME, et al. Myocardial commitment from human pluripotent stem cells: Rapid production of human heart grafts Biomaterials 2016;98;64e78

[3] Lee P, Klos M, Bollensdorff C, et al. Simultaneous voltage and calcium mapping of genetically purified human induced pluripotent stem cell-derived cardiac myocyte monolayers. Circ Res. 2012;110(12):1556-63.

[4] Climent AM, Guillem MS, Fuentes L, et al. Role of atrial tissue remodeling on rotor dynamics: an in vitro study. Am J Physiol Heart Circ Physiol. 2015;309(11):H1964-73

[5] Liberos A, Bueno-Orovio A, Rodrigo M, et al. Balance between sodium and calcium currents underlying chronic atrial fibrillation termination: An in silico inter-subject variability study. Heart Rhythm. 2016. In press

Address for correspondence.

Andreu M. Climent

Laboratorio de órganos y matrices bioartificiales

Instituto de Investigación Sanitaria Gregorio Marañón

Hospital GU Gregorio Marañón. Edificio Materno Infantil.

c〉 O'Donnell 48, 28009, Madrid, Spain

andreu.climent@gmail.com 